
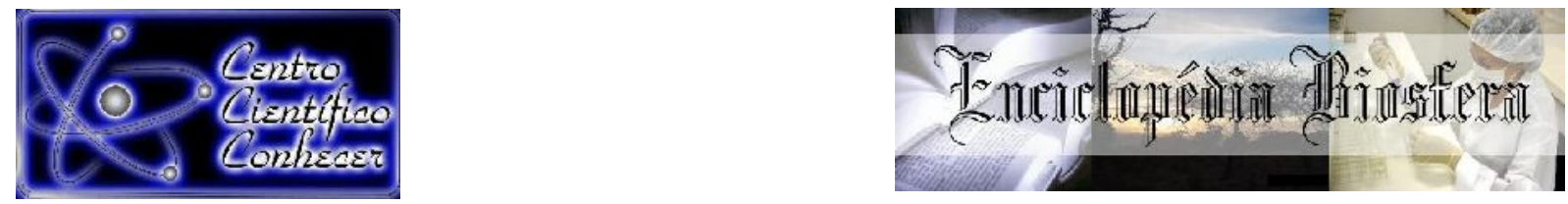

\title{
MASTOFAUNA ENCAMINHADA AO CENTRO DE TRIAGEM DE ANIMAIS SILVESTRES (CETAS) DE CATALÃO, GOIÁS
}

Bruna Rafaella de Almeida Nunes¹, Diogo Baldin Mesquita², Thatiana Martins dos Santos Mesquita ${ }^{3}$

${ }^{1}$ Mestranda em Estudos Ambientais da Universidade Federal de Catalão (UFCAT), Catalão-GO, Brasil

E-mail: brunarafaella@gmail.com

2Médico Veterinário, Coordenador do Centro de Triagem de Animais Silvestres (CETAS), Catalão-GO, Brasil

${ }^{3}$ Bióloga, Agente de Fiscalização Ambiental da Secretaria Municipal de Meio Ambiente de Catalão (SEMMAC), Catalão-GO, Brasil

Recebido em: 15/08/2020 - Aprovado em: 15/09/2020 - Publicado em: 30/09/2020 DOI: 10.18677/EnciBio_2020C26

\begin{abstract}
RESUMO
Os mamíferos desempenham um papel importante na cadeia alimentar regulando a população de outras espécies animais, bem como na reprodução vegetal com a polinização e dispersão de sementes. As populações de espécies silvestres sofrem declínios determinados principalmente pela fragmentação e perda de habitats. O presente estudo identificou e quantificou os mamíferos silvestres encaminhados ao Centro de Triagem de Animais Silvestres (CETAS) de Catalão, considerando a importância da divulgação das informações que podem servir de subsidio para as ações de conservação da biodiversidade. O trabalho foi realizado através de pesquisa documental no banco de dados do CETAS de Catalão, respectivamente no período entre 2016 a 2019. No período analisado foram registrados 260 espécimes de mamíferos, distribuídos em 8 ordens, 14 famílias e 22 espécies. A espécie de mamífero mais abundante foi Didelphis albiventris (gambá-de-orelha-branca). Das espécies registradas Chrysocyon brachyurus (lobo-guará), Lontra longicaudis (lontra-neotropical), Myrmecophaga tridactyla (tamanduá-bandeira) e Priodontes maximus (tatu-canastra) constam na lista de espécies ameaçadas de extinção do ICMBio e IUCN. O município de Catalão apresentou maior número de indivíduos encaminhados, e o Corpo de Bombeiros Militar do Estado de Goiás foi órgão responsável pelo maior número de encaminhamentos. A diversidade e abundância de espécies de mamíferos encaminhados ao CETAS de Catalão, no período analisado demonstra a importância da existência dos CETAS para recebimento dos animais silvestres em conflito com a ocupação do solo pelas atividades do homem.
\end{abstract}

PALAVRAS-CHAVE: Biodiversidade, Conservação, Mamíferos.

\section{MASTOFAUNA DIRECTED TO THE CENTER OF SCREENING OF WILD ANIMALS (CETAS) OF CATALÃO, GOIÁS}

\footnotetext{
ABSTRACT

Mammals play an important role in the food chain by regulating the population of other animal species, as well as in plant reproduction with pollination and seed dispersal. ENCICLOPÉDIA BIOSFERA, Centro Científico Conhecer - Jandaia-GO, v.17 n.33; p. 286 2020
} 
Populations of wild species suffer declines determined mainly by fragmentation and loss of habitats. This study identified and quantified the wild mammals referred to the Centro de Triagem de Animais Silvestres (CETAS) in Catalão, considering the importance of disseminating information that can serve as a subsidy for biodiversity conservation actions. The work was carried out through documentary research in the database of the CETAS of Catalão, respectively in the period between 2016 and 2019. In the period analyzed 260 specimens of mammals were recorded, distributed in 8 orders, 14 families and 22 species. The most abundant mammal species was Didelphis albiventris (whiteeared opossum). Between the registered species Chrysocyon brachyurus (maned-wolf), Lontra longicaudis (neotropical-otter), Myrmecophaga tridactyla (giant-anteater) and Priodontes maximus (giant-armadillo) are on the ICMBio and IUCN endangered species list. The municipality of Catalão presented the highest number of individuals referred, and the Military Fire Department of the State of Goiás was responsible for the highest number of referrals. The diversity and abundance of mammal species forwarded to the CETAS of Catalão, in the period analyzed demonstrates the importance of the existence of the CETAS to receive wild animals in conflict with the occupation of the soil by human activities.

KEYWORDS: Biodiversity, Conservation, Mammals.

\section{INTRODUÇÃO}

O Brasil é um país que apresenta ampla extensão territorial, composta por diferentes paisagens, climas e biomas, tornando um dos países com alta diversidade biológica mundial. A fauna de vertebrados é composta por 8.922 espécies, distribuídas nos seis biomas brasileiros, onde os mamíferos constituem o menor índice de diversidade se comparado aos outros grupos de organismos, apresentando 732 espécies (ICMBIO, 2018).

Os mamíferos de pequeno porte podem disponibilizar informações acerca da sua ecologia com maior facilidade em virtude do grande número de indivíduos. Os mamíferos carnívoros, por sua vez desempenham um importante papel ecológico através do controle de populações, especialmente suas presas, que incluem aves, répteis e pequenos mamíferos, bem como na manutenção dos ecossistemas (CORLETT; HUGHES, 2015). Além disso, possuem capacidade de dispersar sementes de diversas espécies de plantas e atuando na recuperação de áreas florestais (ARÉVALO-SANDI et al., 2018).

As populações de espécies silvestres têm sofrido declínios consideráveis e preocupantes, determinados principalmente pela fragmentação e perda de habitats, bem como pela construção de rodovias, expansão agrícola, desmatamento e o avanço do crescimento urbano em áreas de remanescentes florestais (SCANES, 2018). Apesar dos ambientes naturais sofrerem fragmentações, essas pequenas ilhas remanescentes podem disponibilizar recursos à determinadas espécies, configurando-se como trampolins para os indivíduos. Essa conexão permite a identificação das espécies encontradas nos fragmentos de vegetação, além dos padrões de distribuição e informações relevantes e fundamentais para a implementação de planos de manejo e conservação (CHIARELLO et al., 2008).

Os fragmentos de vegetação como as áreas verdes urbanas podem abrigar diversas espécies de mamíferos que se adaptam as condições impostas pelas cidades e a presença de humanos. Segundo Vilela et al. (2016) as alterações relacionadas a 
luminosidade, umidade, ruídos, temperatura e interação entre espécies provoca a redução e/ou extinção de populações locais, bem como a substituição por espécies mais generalistas.

No Cerrado Goiano, as pastagens cultivadas e as culturas agrícolas são as principais formas de uso da terra na região Sudeste de Goiás (SANO et al., 2008), a perda da biodiversidade pelo desmatamento e consequente diminuição de oferta de alimentação e local de abrigo tem forte impacto nas populações de mamíferos, sobretudo de médio e grande porte. Além das atividades de pecuária e agricultura, a atividade de mineração no entorno de Catalão transforma as paisagens do entorno desde a década de 1970 (BRANCO FILHO, 2019), afetando os recursos hídricos, o solo, a fauna e a flora.

Com a intensificação dos impactos ocasionados pelas ações antrópicas em ambientes naturais, a fauna silvestre desloca-se em busca de habitats que disponibilizem condições necessárias para a sua sobrevivência. Durante sua movimentação, os animais silvestres deparam-se com as áreas urbanas, proporcionando benefícios, como também gerando situações de conflitos com a sociedade (VILELA et al., 2016). Ainda segundo os autores, os animais silvestres buscam abrigo e alimentação nas áreas urbanas, e assim adentram em residências e comércios, causando desconforto na população humana.

A ausência de predadores e disponibilidade de recursos ocasiona a proliferação de espécies silvestres em áreas urbanas, e ficam expostas a contaminação de patógenos, agressões e perseguições por animais domésticos, vítimas de maus tratos, atropelados, bem como capturados e mantidos ilegalmente em cativeiro. Nesse intuito, os Centros de Triagem de Animais Silvestres (CETAS) são empreendimentos responsáveis pelo recebimento, reabilitação e destinação da fauna silvestre encaminhadas por órgãos competentes e pela comunidade (BRASIL, 2020).

Tendo em vista a importância da divulgação das informações que podem servir de subsidio para as ações de conservação da biodiversidade no Estado de Goiás, o presente estudo tem como objetivo identificar e quantificar os mamíferos silvestres encaminhados ao Centro de Triagem de Animais Silvestres (CETAS) de Catalão, no período entre 2016 a 2019, bem como a origem, procedência, condições e sazonalidade da entrada dos animais.

\section{MATERIAL E MÉTODOS}

A metodologia utilizada para a realização do presente estudo foi a análise documental, definida como pesquisa aos documentos que não foram analisados e sistematizados (KRIPKA, 2015). A pesquisa documental foi realizada no Centro de Triagem de Animais Silvestres (CETAS) de Catalão, através de consultas no banco de dados dos anos de 2016 a 2019, período em que os registros estão disponíveis.

O município de Catalão está localizado no Sudeste de Goiás, entre os

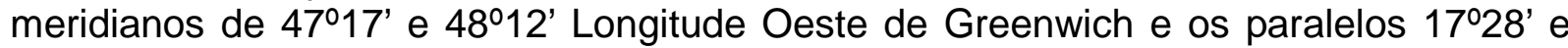
1830' Latitude Sul, abrangendo uma área de $3.817,927 \mathrm{~km}^{2}$ e estimativa populacional de 108.823 pessoas (IBGE, 2019). Apresenta a vegetação característica de Cerrado, com clima tropical, estações definidas de seca e chuva, além da predominância de planaltos e chapadas, cujas características são ideais para as atividades agropecuárias e de mineração (SILVA; ROSA, 2019).

Para a realização do presente estudo, foi realizada uma pesquisa documental no 
banco de dados do CETAS de Catalão, considerando somente os mamíferos silvestres encaminhados no período entre 2016 a 2019. Além disso, foram analisados os locais de origem, os responsáveis pela entrega, categoria (apreensão, resgate e entrega espontânea), condições físico/clínicas e sazonalidade. Os dados coletados foram registrados em planilha do Microsoft Excel 2019, e tratados quantitativamente através de gráficos e tabelas.

Informações acerca da identificação, nomenclatura científica, distribuição geográfica e status de conservação das espécies de mamíferos silvestres brasileiros foram consultadas no Livro Vermelho da Fauna Brasileira Ameaçada de Extinção, Volume II - Mamíferos, publicado pelo Instituto Chico Mendes de Conservação da Biodiversidade (ICMBIO, 2018), bem como na Lista Vermelha da União Internacional para a Conservação da Natureza e dos Recursos Naturais (IUCN, 2020).

\section{RESULTADOS E DISCUSSÃO}

Através das análises dos registros de mamíferos silvestres encaminhados ao CETAS de Catalão no período entre 2016 a 2019, foram identificados 260 espécimes, distribuídos em 8 ordens, 14 famílias e 22 espécies (quadro 1), de hábitos carnívoros, insetívoros, herbívoros e frugívoros.

QUADRO 1- Lista das espécies de mamíferos registrados no CETAS de Catalão, entre os anos de 2016 a 2019.

\begin{tabular}{|c|c|c|c|}
\hline FAMÍLIA & ESPÉCIES & NOME COMUM & $\begin{array}{c}\text { No } \\
\text { INDIVíDUOS }\end{array}$ \\
\hline Didelphidae & Didelphis albiventris (Lund, 1840) & Gambá-de-orelha-branca & 91 \\
\hline Myrmecophagidae & Myrmecophaga tridactyla (Linnaeus, 1758) & Tamanduá-bandeira & 40 \\
\hline Callithichidae & Callithrix penicillata (E. Geoffroy, 1812) & Sagui-de-tufo-preto & 26 \\
\hline Eretizontidae & Coendou prehensilis (Linnaeus, 1758) & Ouriço-cacheiro & 24 \\
\hline Myrmecophagidae & Tamandua tetradactyla (Linnaeus, 1758) & Tamanduá-mirim & 21 \\
\hline Dasypodidae & Dasypus novemcinctus (Linnaeus, 1758) & Tatu-galinha & 9 \\
\hline Canidae & Cerdocyon thous (Linnaeus, 1766) & Cachorro-do-mato & 8 \\
\hline Dasypodidae & Euphractus sexcinctus (Linnaeus, 1758) & Tatu-peba & 6 \\
\hline Canidae & Lycalopex vetulus (Lund, 1842) & Raposa-do-campo & 6 \\
\hline Atelidae & Alouatta caraya (Humboldt, 1812) & Bugio-preto & 5 \\
\hline Caviidae & Hydrocheorus hydrochaeris (Linnaeus, 1766) & Capivara & 5 \\
\hline Cervidae & Mazama gouazoubira (G. Fischer, 1814) & Veado-catingueiro & 5 \\
\hline Procyonidae & Nasua nasua (Linneaus, 1766) & Quati & 3 \\
\hline Canidae & Chrysocyon brachyurus (Illiger, 1815) & Lobo-guará & 2 \\
\hline Didelphidae & Gracilianus agilis (Burmeister, 1854) & Cuíca-graciosa & 2 \\
\hline Dasypodidae & Cabassous unicinctus (Linnaeus, 1758) & Tatu-do-rabo-mole & 1 \\
\hline Caviidae & Cavia aperea (Exerleben, 1777) & Preá & 1 \\
\hline Felidae & Herpailurus yagouaroundi (E. Geoffroy, 1803) & Gato-mourisco & 1 \\
\hline Mustelidae & Lontra longicaudis (Olfers, 1818) & Lontra-neotropical & 1 \\
\hline Dasypodidae & Priodontes maximus (Kerr, 1792) & Tatu-canastra & 1 \\
\hline Cebidae & Sapajus libidinosus (Spix, 1823) & Macaco-prego & 1 \\
\hline NI & NI & Morcego & 1 \\
\hline
\end{tabular}

Fonte: CETAS (2016;2017;2018;2019).

Quanto a ordem dos mamíferos, a Didelphimorphia apresentou maior abundância $(n=93)$, representada por duas espécies de marsupiais, Didelphis albiventris e Gracilianus agilis, seguido da Ordem Pilosa (tamanduás, $n=61$ ), Primatas ENCICLOPÉDIA BIOSFERA, Centro Científico Conhecer - Jandaia-GO, v.17 n.33; p. 289 
(macacos, $n=32$ ) e Rodentia (roedores, $n=30$ ). A ordem Chiroptera (morcegos) foi representada por apenas um espécime não identificado.

A espécie de mamífero que apresentou maior número de indivíduos foi Didelphis albiventris $(n=91)$ correspondendo a $35 \%$ de abundância. Tal abundância corrobora com a pesquisa desenvolvida por Capaverde-Jr et al. (2018) e Avelar et al. (2015), no CETAS do IBAMA, em Goiânia (GO), onde os autores relatam o recebimento de filhotes da espécie. Em seguida Myrmecophaga tridactyla (15\%), Callithrix penicillata (10\%), Coendou prehensilis $(9,2 \%)$ e Tamandua tetradactyla (8\%). A abundância de tais espécies corrobora com os trabalhos desenvolvidos em outras regiões do País, como Dias e Bocchiglieri (2015) em Sergipe, e Nascimento et al. (2016) no Acre.

Espécies generalistas como Didelphis albiventris, popularmente conhecidos como gambá-de-orelha-branca, são beneficiadas por se deslocarem em áreas abertas e fechadas em busca de recursos, tanto no interior do fragmento como na borda (MALCOM, 1994). Além disso, podem ainda se beneficiar com a fragmentação, pois seu padrão amplo de deslocamento permite a troca de fluxo genético, contribuindo para o aumento e densidade das espécies que se adaptam as ações e intervenções antrópicas (WOLFF et al., 1997).

Dentre as vinte e duas espécies de mamíferos identificadas nesse estudo, quatro estão enquadradas em 2 categorias de ameaça a extinção, correspondente a Vulnerável (VU) pelo ICMBio (2018), Vulnerável (VU) e Quase Ameaçada (NT) pela IUCN. Os critérios utilizados para determinar o nível de ameaça de uma espécie estão intimamente ligados a redução da população, distribuição geográfica restrita, populações pequenas com declínio, fragmentação e distribuição muito restrita, além da análise quantitativa do risco de extinção.

As espécies de xenartras Priodontes maximus (tatu-canastra) e Myrmecophaga tridactyla (tamanduá-bandeira) são classificadas como vulneráveis de extinção, e Lontra longicaudis (Iontra-neotropical) como quase ameaçada, de acordo com os critérios do ICMBIO e IUCN. No entanto, Chrysocyon brachyurus (lobo-guará) está listado como quase ameaçado pela IUCN, enquanto o ICMBio (2018) avaliou a espécie como vulnerável de extinção, uma vez que as principais ameaças sobre a espécie são a perda de hábitat, principalmente no Bioma Cerrado, perda de indivíduos por conflitos com humanos e perda de indivíduos nas rodovias, vítimas de atropelamentos.

No que diz respeito a procedência dos mamíferos encaminhados ao CETAS de Catalão, foram identificados treze municípios de origem no Estado de Goiás, além de animais provenientes da Rodovia GO-210, do município de Uberlândia (MG), e os de locais de origem não identificado $(\mathrm{NI})$. O município de Catalão apresentou maior número de indivíduos $(n=175)$ correspondendo a $67,3 \%$ dos locais de origem, seguido dos municípios goianos de Davinópolis $(5,3 \%)$, Ouvidor $(3,8 \%)$, Cumari $(3,4 \%)$ e Goiandira (3\%), conforme Figura 1. Os mamíferos de origem não identificada contabilizaram dezenove indivíduos, e os demais locais apresentaram de um a seis indivíduos cada. 
FIGURA 1 Origem da mastofauna encaminhada ao CETAS de Catalão, de 2016 a 2019.

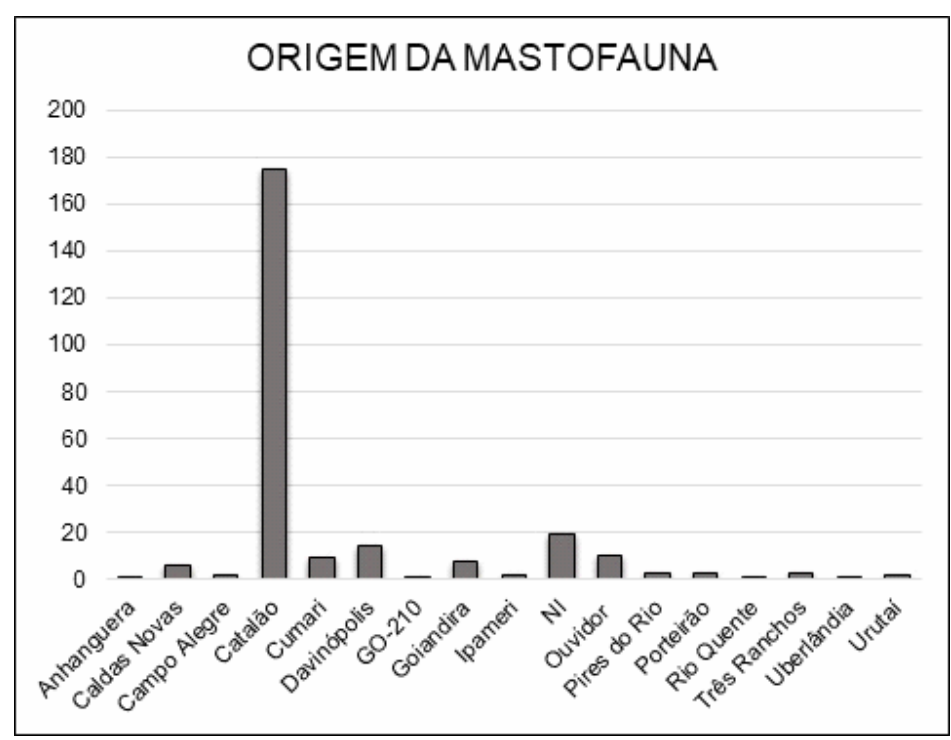

Fonte: CETAS (2016;2017;2018;2019).

Ocorrência de animais silvestres em áreas urbanizadas tornou-se cada vez mais frequente, uma vez que a fragmentação e destruição dos habitats naturais conduzem as espécies a buscarem abrigo e alimento nos centros urbanos. Os mamíferos registrados no CETAS nos anos de 2016 a 2019 foram resgatados e encaminhados pelos Batalhões do Corpo de Bombeiros Militar instalados nos municípios de Catalão, Ipameri e também de Pires do Rio representando $69,6 \%$ dos encaminhamentos. As entregas espontâneas realizadas pela comunidade corresponderam a $23 \%$, os encaminhados pela Secretaria Municipal de Meio Ambiente de Catalão (SEMMAC) 4,3\%, Polícia Militar Ambiental (PMA) 2,3\%. A Universidade Federal de Uberlândia (UFU), no Estado de Minas Gerais, e Secretaria de Estado de Meio Ambiente e Desenvolvimento Sustentável (SEMAD) corresponderam a 0,4\% dos encaminhamentos, como mostra a Figura 2.

FIGURA 2 Destinatários da mastofauna encaminhada ao CETAS de Catalão, de 2016 a 2019.

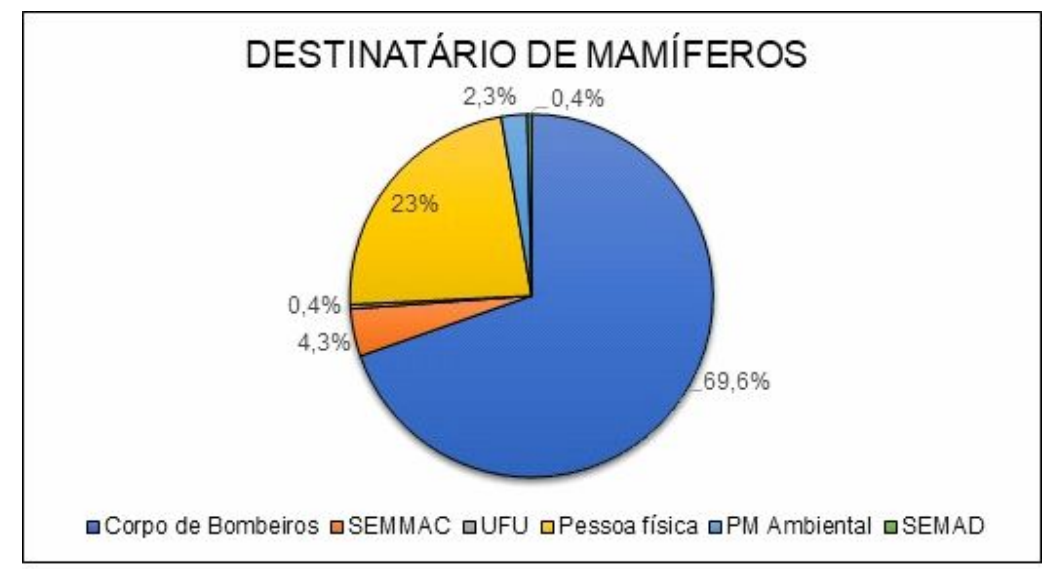

Fonte: CETAS (2016;2017;2018;2019). 
Ações antrópicas nos habitats naturais direcionam a fauna silvestre para áreas urbanas, e que por vezes causa medo e pânico na população, sendo necessária a intervenção de profissionais habilitados para o resgate de animais (VILELA et al., 2016). Dessa forma, o Corpo de Bombeiros Militar de Catalão realiza grande parte dos resgates envolvendo mamíferos silvestres de pequeno, médio e grande porte, que estão circulando em vias públicas, adentraram em residências, comércios, vítimas de atropelamento entre outras situações em áreas urbanas e rurais.

Entre as categorias de entrega dos mamíferos ao CETAS de Catalão destacamse no presente estudo a apreensão, quando indivíduos da fauna silvestre são apreendidos e recolhidos em situação ilegal, a entrega espontânea, realizada voluntariamente por munícipe, e os resgates realizados em grande parte pelo Corpo de Bombeiros Militar, bem como pelo CETAS e o Departamento de Fiscalização Ambiental (DFA) da SEMMAC.

Os registros de mamíferos apresentados na Figura 3 mostram a maior frequência de resgates $(73,8 \%)$, seguido por entrega voluntária $(25,7 \%)$ e apreensão $(0,5 \%)$, sendo esses resultados semelhantes ao trabalho realizado por Silva (2015) em Salvador, no Estado da Bahia.

FIGURA 3 Categoria de entrega dos mamíferos ao CETAS de Catalão, de 2016 a 2019.

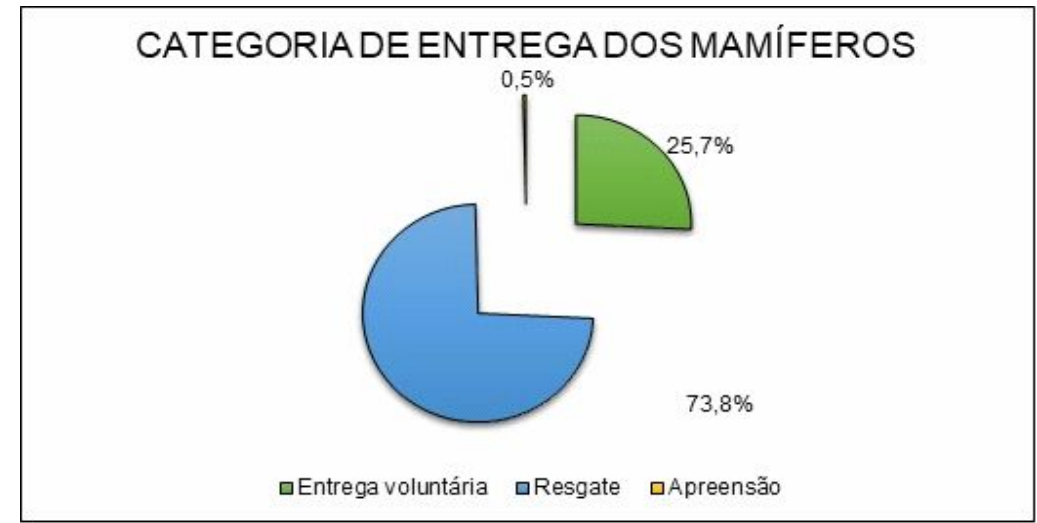

Fonte: CETAS $(2016 ; 2017 ; 2018 ; 2019)$.

São diversas as condições clínicas em que os animais são encaminhados ao CETAS como por exemplo, atacados e feridos por animais domésticos, vítimas de maus tratos, perfurados por bala, queimados entre outras. Avelar et al. (2015) relatam que a maioria dos mamíferos encaminhados ao CETAS/IBAMA em Goiânia chegam debilitados, apresentando fratura nos membros, eletrocutados ou atropelados. Diante disso, é importante que a captura do animal seja realizada de forma adequada, visando a integridade física e clínica do animal, como também do responsável pelo manejo, evitando o agravamento de quadro clínico.

Os mamíferos saudáveis, e aptos a reintrodução em seu habitat natural, corresponderam a 70\% das condições analisadas, conforme a Figura 4. Os animais que apresentaram ferimentos como lesões e fraturas $21 \%$ e os debilitados $8 \%$. Indivíduos que não resistiram aos ferimentos e vieram a óbito, durante o transporte para o CETAS, 
corresponderam a 1\%, representados por Callithrix penicillata (sagui-de-tufo-preto) e Priodontes maximus (tatu-canastra), vítimas de atropelamento com veículo automotor.

FIGURA 4 Condições clínicas dos mamíferos encaminhados ao CETAS de Catalão, de 2016 a 2019.
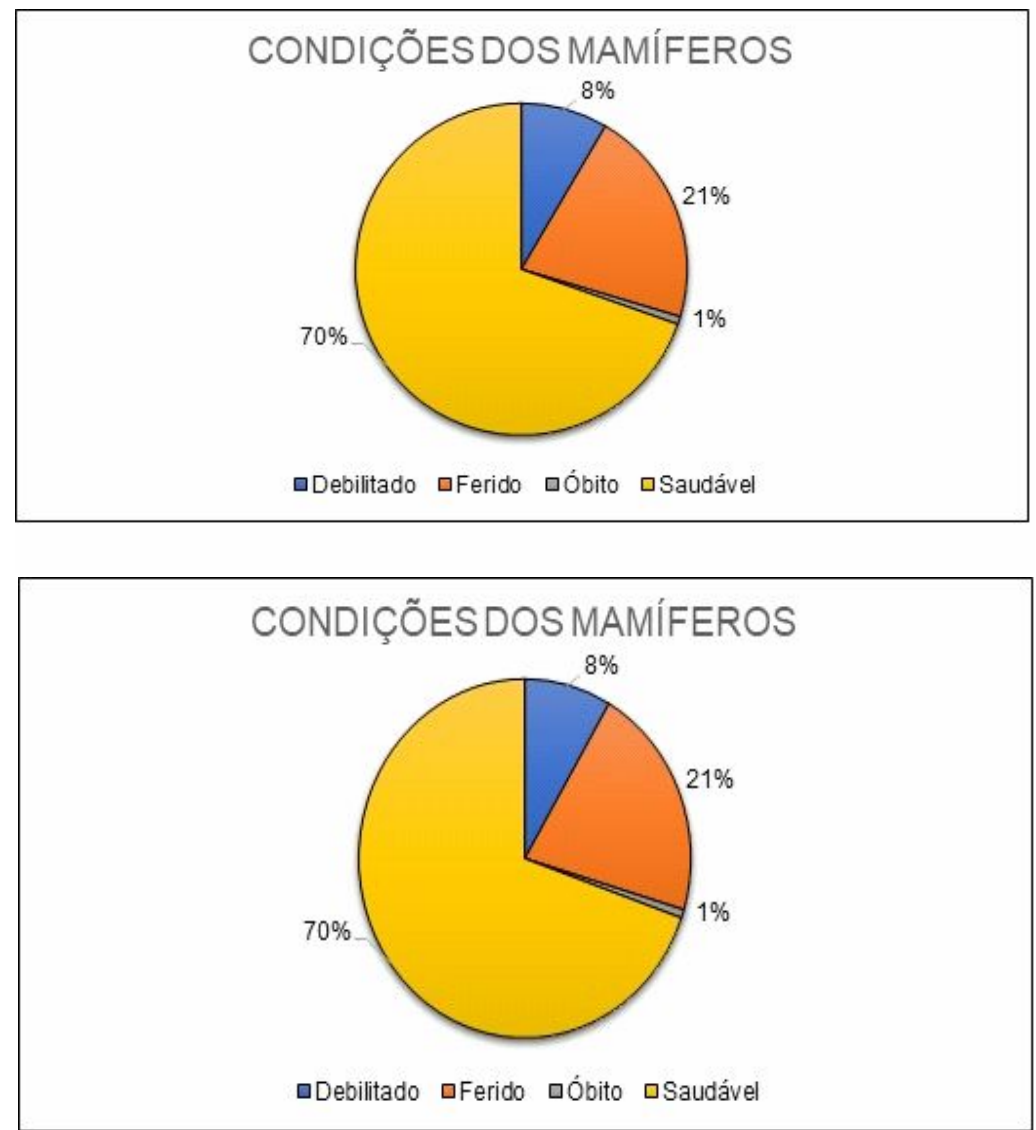

Fonte: CETAS $(2016 ; 2017 ; 2018 ; 2019)$.

No que se refere ao período de encaminhamento de mamíferos ao CETAS de Catalão, no ano de 2016 foram registrados 47 mamíferos, em 2017 foram registrados 67, 2018 registrou 70 e o ano de 2019 registrou 76 mamíferos. Quanto aos meses de maior ocorrência, os respectivos meses foram novembro, outubro, dezembro, abril e setembro. Em contraste, o período com menor ocorrência foram os meses de junho, agosto, janeiro e julho (Figura 5). 
FIGURA 5 Sazonalidade dos mamíferos encaminhados ao CETAS de Catalão, de 2016 a 2019.

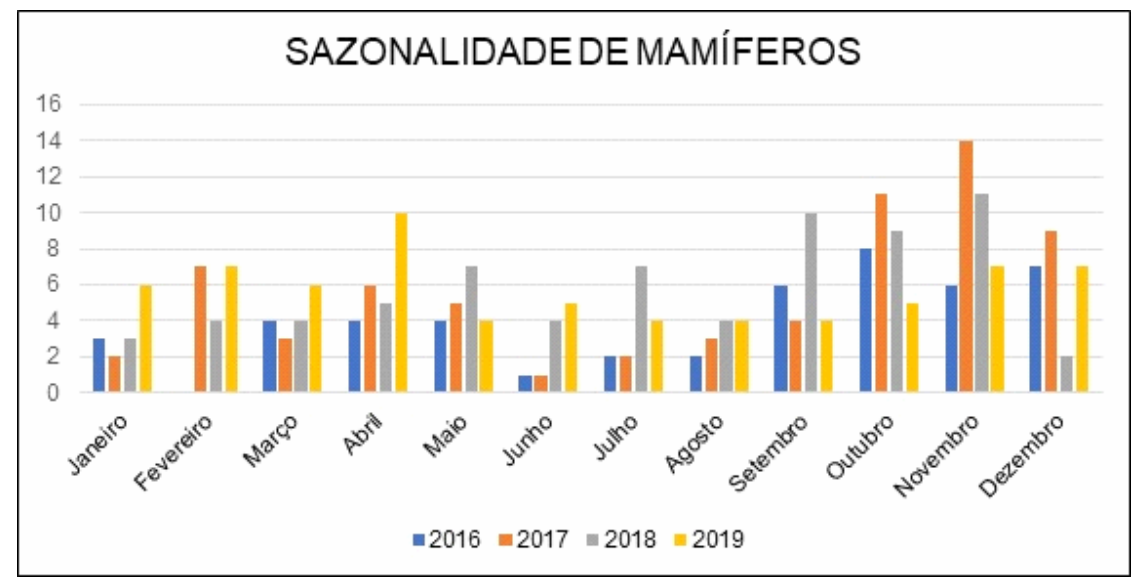

Fonte: CETAS $(2016 ; 2017 ; 2018 ; 2019)$.

Foi possível observar maior ocorrência de encaminhamento de mamíferos ao CETAS de Catalão no período chuvoso, correspondendo aos meses de outubro e novembro. Nesse período há uma maior disponibilidade de alimentos, lançamento foliar de espécies arbóreas e frutificação, deslocamento de animais, além de ser o período reprodutivo de espécies, como por exemplo de Didelphis albiventris (SÁ, 2014).

Tais fatores associados a destinação inadequada de resíduos, desmatamento e degradação ambiental nas áreas periféricas e estradas vicinais no entorno do município sujeita a aproximação dos animais com o meio urbano. As áreas verdes do município podem ainda se constituir como habitat para algumas espécies que se adaptam ao meio antrópico, entretanto tornam-se vulneráveis a doenças ocasionadas por animais domésticos e agressões pelos humanos.

Os CETAS fornecem informações sobre a fauna local e/ou regional e contribuem para o conhecimento das espécies de animais silvestres. Dessa forma, estudos de levantamento de fauna fornecem subsídios para futuras pesquisas, ações fiscalizatórias e educativas no Sudeste Goiano, tanto em nível governamental, em ações como o Plano de Ação Nacional para Conservação de Espécies Ameaçadas de Extinção - PAN, mas também de investimentos privados na conservação da biodiversidade, especialmente do bioma Cerrado.

\section{CONCLUSÃO}

O presente estudo apresentou uma variedade de mamíferos de pequeno, médio e grande porte encaminhados ao CETAS de Catalão no período entre 2016 a 2019. As espécies Didelphis albiventris, Myrmecophaga tridactyla, Callithrix penicillata, Coendou prehensilis e Tamandua tetradactyla obtiveram maior número de espécimes. Ainda, foram identificados registros de espécies classificadas como vulneráveis, quase ameaçada e ameaçada de extinção como Priodontes maximus, Lontra longicaudis, Myrmecophaga tridactyla e Chrysocyon brachyurus.

Grande parte dos mamíferos silvestres encaminhados são provenientes de Catalão e resgatados pelo Corpo de Bombeiros Militar, o que ressalta a importância do 
CETAS no município e na região Sudeste de Goiás para a destinação adequada da fauna silvestre.

O período analisado mostrou um crescimento anual de encaminhamentos, principalmente na transição das estações seca para a chuvosa. Tal fato pode estar relacionado a disponibilidade de alimentos e período reprodutivo de algumas espécies, provocando o deslocamento em ambientes urbanos. Estudos de monitoramento da fauna nas áreas verdes urbanas e do entorno do município são importantes para fornecer dados acerca das espécies e como elas utilizam esses fragmentos.

Diante do exposto, os resultados dessa pesquisa podem auxiliar na elaboração e execução de ações voltadas à educação ambiental, no intuito de sensibilizar a comunidade sobre a importância, a conservação e preservação da fauna silvestre, bem como o seu papel ecológico.

\section{REFERÊNCIAS}

ARÉVALO-SANDI, A.; BOBROWIEC, P. E. D.; CHUMA, V. J. U. R.; NORRIS, D. Diversity of terrestrial mammals seed dispersers along a lowland Amazon Forest $\begin{array}{lllllll}\text { regrowth } & \text { gradient. Plos } & \text { One. } & \text { v. } & 13 & \text { (3). }\end{array}$ Disponível em https://doi.org/10.1371/journal.pone.0193752

AVELAR, E. R.; SILVA; R.; BAPTISTA; L. A. L. Ameaças à Sobrevivência de Animais Silvestres no Estado de Goiás. Uniciências. v.19, n. 02., p. 132-140. 2015. Disponível em:

https://pdfs.semanticscholar.org/8b97/b9b17189844a49b6d7e7857a914e0cc5148f.pdf? ga $=2.58570154 .1610209181 .1597539162-1837281483.1589033748$

BRASIL- Ministério do Meio Ambiente. Biodiversidade. Acesso em 08 jul. 2020. Disponível em: https://www.mma.gov.br/mma-em-numeros/biodiversidade

BRANCO FILHO, T. C.T. O caso da exploração do nióbio em Catalão. Lumen Juris, Rio de Janeiro, 404 p. 2019

CAPAVERDE-JR, U. D.; LOPES, M. S., ALMEIDA, N. C. V., ALMEIDA, F. Z. P., PATHEK, D. B. Animais recolhidos pela Companhia Independente de Policiamento Ambiental Monte Roraima na área urbana de Boa Vista, Amazônia Brasileira. Revista Biota Amazônia. v. 8. n. 1, p 43-48. 2018. Disponível em http://dx.doi.org/10.18561/2179-5746/biotaamazonia.v8n1p43-48

CHIARELLO, A. G.; AGUIAR, L. M. S.; CERQUEIRA, R.; MELO, F. R.; RODRIGUES, F. $\mathrm{H}$.; SILVA, V. M. Livro Vermelho da Fauna Brasileira Ameaçadas de Extinção, Volume 2: Mamíferos 1. Editora MMA - Ministério do Meio Ambiente. 203 p. 2008.

CORLETT, R. T.; HUGHES, A. Mammals in forest ecosystems. In: The Routledge Handbook of Forest Ecology. PEH, K. S.-H., CORLETT, R.T.; BERGERON, Y. (eds.) Routledge, Oxford, UK, p. 264-278. 2015. Disponível em: https://www.researchgate.net/publication/287998935_Mammals_in_forest_ecosystems 
CUARÓN, A. D. A global perspective on habitat disturbance and tropical rainforest mammals. Conservation Biology, v. 14, n. 6, p. 1574-1579. 2000. Disponível em: https://www.researchgate.net/publication/233967863_A_global_perspective_on_habitat disturbance_and_tropical_rainforest_mammals

DIAS, D. M.; BOCCCHIGLIERI, A. Mamíferos silvestres recolhidos em áreas urbanas de Sergipe, Nordeste do Brasil. Revista Nordestina de Zoologia. v. 9, n.1, p. 1-15. 2015. Disponível em: https://www.academia.edu/38158345/Mam\%C3\%ADferos_silvestres_recolhidos_em_\% C3\%A1reas_urbanas_de_Sergipe_nordeste_do_Brasil_pdf

IBGE - Instituto Brasileiro de Geografia e Estatística . Cidades e estados. Catalão. Disponível em: https://www.ibge.gov.br/cidades-e-estados/go/catalao.html

ICMBIO - Instituto Chico Mendes de Conservação a Biodiversidade. Livro Vermelho da Fauna Brasileira Ameaçada de Extinção: Volume 1. 1aㅡ ed. 492 p. Brasília, DF: ICMBio/MMA, 2018.

IUCN - International Union for Conservation of Nature. The IUCN Red List of Threatened Species. Version 2020-1. Acesso em 08 jul. 2020. Disponível em: https://www.iucnredlist.org/

KRIPKA, R. M. L.; SCHELLER, M.; BONOTTO, D. L. Pesquisa documental na pesquisa qualitativa: conceitos e caracterização. Revista de Investigaciones. v. 14. n. 2, 2015. Disponível em: https://www.researchgate.net/publication/280924900_Pesquisa_Documental_considerac oes_sobre_conceitos_e_caracteristicas_na_Pesquisa_Qualitativa_Documentary_Resea rch_consideration_of_concepts_and_features_on_Qualitative_Research

MALCOM, JR. Forest structure and the abundance and the diversity of Neotropical small mammals. In: Forest Canopies (Lowman, MD e NM Nadkarni, eds.). Academic Press, San Diego, p. 179-197.1994. Disponível em: https://www.semanticscholar.org/paper/Forest-structure-and-the-abundance-anddiversity-of-Malcolm-Lowman/013991d23d3b220f07a7e25ae6ec06aba143ea32

SÁ, E. F. G. G., MENDES, D. A. S., CHEUNG, K. C. Levantamento e caracterização de Didelphis albiventris (Lund 1840) em fragmentos de Cerrado na Fazenda Cervinho, Bandeirantes, MS, Brasil. Multitemas, n. 45, p. 83-102. 2014. Disponível em: https://www.multitemas.ucdb.br/multitemas/article/view/237/282

SANO, E.; ROSA, R. R. T.; BRITO, J. L. S.; FERREIRA, L. G. Mapeamento semi detalhado do uso da terra do Bioma Cerrado. Pesquisa Agropecuária Brasileira, v. 43, n. 1 , 2008.2 Disponível em: https://www.researchgate.net/publication/250030761_Mapeamento_semidetalhado_do_ uso_da_terra_do_Bioma_Cerrado 
SCANES, C. G. Human activity and habitat loss: Destruction, Fragmentation, and Degradation. In: Animals ans Human Society, p. 451-482. 2018.

SILVA, A. S.; ROSA, R. Mapa de capacidade e potencial do uso da terra do município de Catalão (GO). Caderno de Geografia, v. 29, n. 59, 2019. Disponível em: http://periodicos.pucminas.br/index.php/geografia/article/view/19522.

https://doi.org/10.5752/P.2318-2962.2019v29n59p954

VILELA, D.; TEIXEIRA, C.; HORTA, C.; LOURA, G. R.; SILVA, M. M. Gestão de conflitos com animais silvestres em centros urbanos. Belo Horizonte, 60 p. 2016.

WOLFF, J.; SCHAUBER, E. M.; EDGE, D. W. Effects of habitat loss and fragmentation on the behavior and demography of Gray-tailed volves. Conservation Biology, v. 11, n.4, p. 945-956. 1997. Disponível em: https://www.jstor.org/stable/2387329 